\title{
Transient vocal cord deformity caused by a laryngeal mask airway device during flexible fibreoptic bronchoscopy
}

\author{
Alex Blais, MD • Richard N. Merchant, MD • \\ Stephen P. Blackie, MD
}

Published online: 19 April 2012

(C) Canadian Anesthesiologists' Society 2012

\section{To the Editor,}

Both anesthesiologists and respirologists commonly perform flexible fibreoptic bronchoscopy. At our institution, an anesthesiologist attends all flexible bronchoscopies that involve endobronchial ultrasound (EBUS), and a laryngeal mask airway device is generally employed for airway protection and introduction of the flexible scope. The use of laryngeal mask airway devices for flexible bronchoscopy is well established as it allows an unobstructed view of the larynx and access to the lower respiratory tract while maintaining airway control. ${ }^{1,2}$

A 75-yr-old female with significant smoking history and a solitary pulmonary nodule presented for diagnostic bronchoscopy and EBUS. The patient's medical history was otherwise notable for a laparoscopic sigmoid colon resection one month prior. Her airway was topicalized with $2-4 \%$ lidocaine via gargle and an atomizing device, and sedation was achieved with propofol and remifentanil infusions. A size 3.5 air- $\mathrm{Q}^{\mathrm{TM}}$ laryngeal mask airway device (Cookgas, Saint Louis, MO, USA) was placed without difficulty. During introduction of the flexible bronchoscope, we noted a significant abnormality of the patient's

A. Blais, MD $(\bowtie)$

Department of Anesthesiology, Pharmacology and Therapeutics, University of British Columbia, Vancouver, BC, Canada e-mail: alexblais@gmail.com

\section{R. N. Merchant, MD}

Department of Anesthesiology, Royal Columbian Hospital, New Westminster, BC, Canada

S. P. Blackie, MD

Division of Respiratory and Critical Care Medicine, Royal Columbian Hospital, New Westminster, BC, Canada left vocal cord and mild bowing of the right (Fig. 1). Our initial suspicion of laryngeal trauma related to the patient's recent anesthetic was disproven when a minor adjustment of the laryngeal mask airway device resolved the deformity (Fig. 2). Normal vocal cord apposition during phonation was subsequently documented, and the patient did not suffer from hoarseness or other dysphonia following the procedure.

Although laryngeal mask airway devices are designed to seat within the hypopharynx, Brimacombe and Berry have pointed out that we lack a detailed understanding of their mechanical impact on pharyngeal structures. ${ }^{3}$ A review of the literature suggests that the overall anatomic

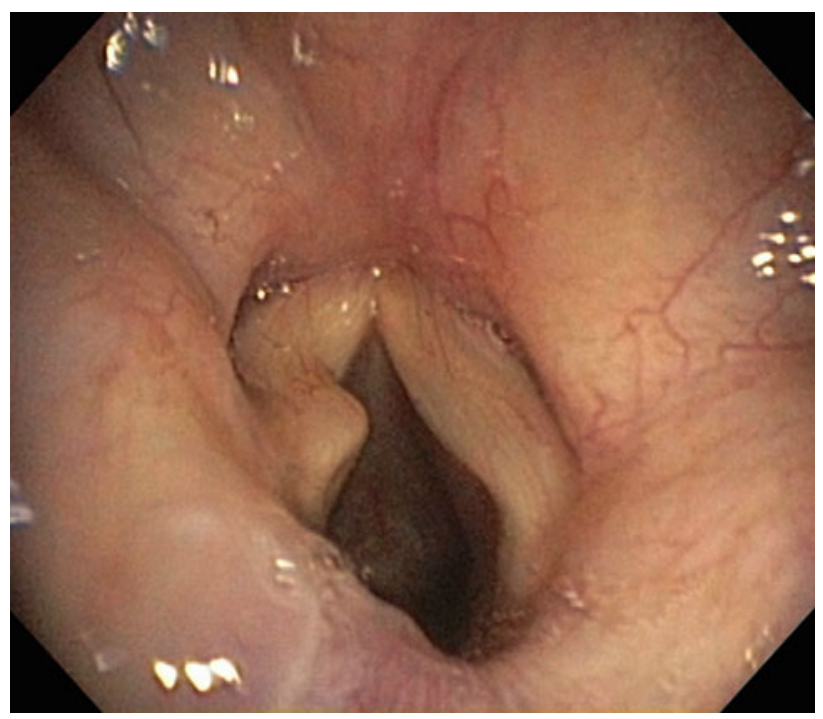

Fig. 1 Visible left vocal cord deformity and mild bowing of the right vocal cord during diagnostic fibreoptic bronchoscopy performed through a laryngeal mask airway device 


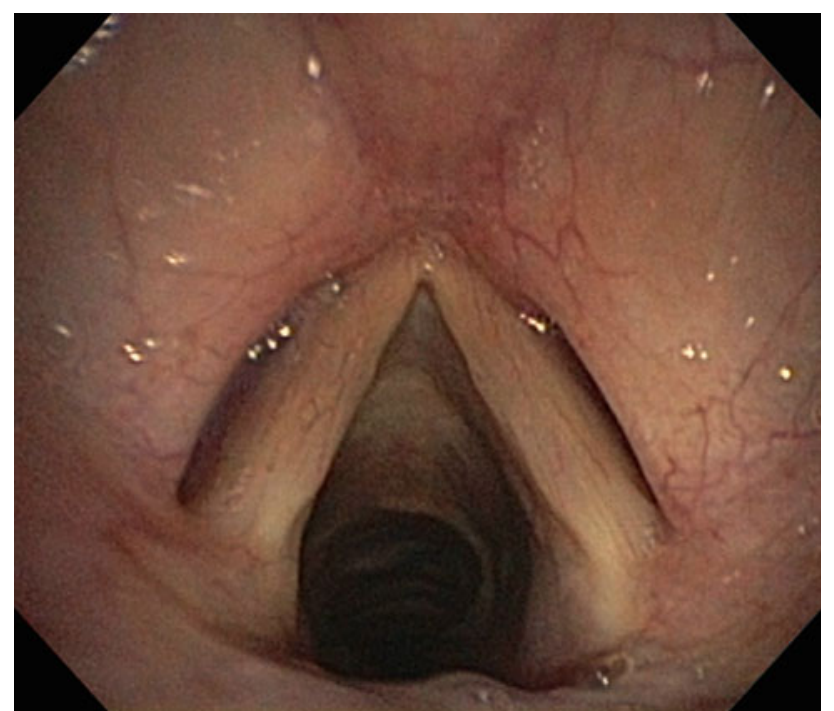

Fig. 2 Resolution of vocal cord deformity following a slight retraction of the laryngeal mask airway device

implications of these devices remain relatively unexplored. We report bronchoscopic evidence of transient vocal cord distortion attributed to mechanical force exerted by a supraglottic device. Our experience suggests that mechanical displacement should be ruled out whenever a vocal cord deformity is identified with flexible bronchoscopy through a laryngeal mask airway device.
Disclosures No funding supported this work.

Competing interests None declared.

\section{References}

1. Brimacombe J, Tucker P, Simons $S$. The laryngeal mask airway for awake diagnostic bronchoscopy. A retrospective study of 200 consecutive patients. Eur J Anaesthesiol 1995; 12: 357-61.

2. Birmingham B, Mentzer SJ, Body SC. Laryngeal mask airway for therapeutic fiberoptic bronchoscopic procedures. J Cardiothorac Vasc Anesth 1996; 10: 519-20.

3. Brimacombe J, Berry A. The laryngeal mask airway - anatomical and physiological implications. Acta Anaesthesiol Scand 1996; 40: 201-9. 